The Annals of Applied Probability

2002, Vol. 12, No. 3, 860-889

\title{
FLUID LIMITS OF STRING VALUED MARKOV PROCESSES ${ }^{1}$
}

\author{
By JeAn-François Dantzer and Philippe Robert \\ Université de Versailler-St-Quentin and INRIA \\ À la mémoire de Vincent Dumas
}

\begin{abstract}
The stability properties of the bandwidth allocation algorithm first fit are analyzed for the distributions concentrated on three sizes for the requests. We give the explicit expression of the ergodicity condition of this model; it involves a quadratic functional of the input parameters. The stochastic processes describing these systems are string valued Markov processes. The notion of a smooth random state is introduced. Starting from a smooth random state the fluid limits of the process can be investigated. The fluid limits of interest are random dynamical systems in $\mathbb{R}^{2}$ which are products of random $2 \times 2$ matrices.
\end{abstract}

\section{CONTENTS}

1. Introduction

2. Fluid limits

3. The string valued Markov process

4. When the natural condition is sufficient for ergodicity

5. Smoothing the initial state

6. A random dynamical system in $\mathbb{R}_{+}^{2}$

7. Ergodicity

8. Transience

Appendix

1. Introduction. The model we consider here is a simplified description of a bandwidth allocation scheme, that is, the allocation of different streams of messages in a communication network. The arriving messages are of different nature; to be transmitted they require different throughputs, that is, variable portions of the offered bandwidth $C$ of the network. The sum of throughputs required by the messages being transmitted at a given time must be less than $C$. If they are not being transmitted, the messages are stored in an infinite buffer in their order of arrival. When a message has finished its transmission, messages in the queue can be transmitted if there is enough room in the network; that is, if the

Received December 2000; revised February 2002.

${ }^{1}$ Supported in part by IST Programme of EU Contract IST-1999-14186 (ALCOM-FT).

AMS 2000 subject classifications. Primary 60K25, 90B12; secondary 60J75, 90B22.

Key words and phrases. Bin packing algorithms, dynamic bin-packing, ergodicity, fluid limits, multiclass queueing systems, bandwidth allocation. 


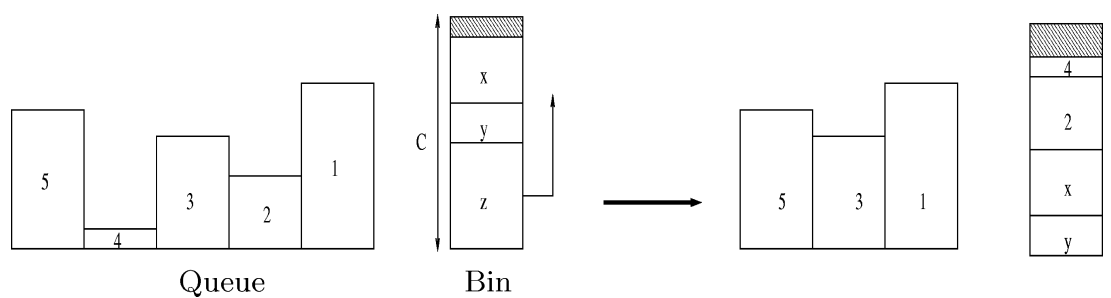

FIG. 1. A departure for the First Fit algorithm.

unused bandwidth is large enough. The allocation algorithm considered here is the First Fit algorithm: A message in the queue is allocated if its throughput is less than the available bandwidth at that time and none of the other messages arrived before it in the queue can be transmitted.

For convenience, bin packing terminology shall be used: The network is a bin of size $C$, messages are items and the bandwidth required by a message is the size of the item. See Coffman and Lueker [6] for a presentation of bin packing problems. Items have the same distribution as some random variable $S_{1}$. A stream of such items arrives at rate $\lambda$ at the bin and each item requires a service of mean 1 . In this setting the First Fit algorithm can be described as follows: The sum of the items in the bin is less than $C$, the size of the bin. Following every event (arrival or departure), the queue is scanned from the beginning in search of an item whose size is smaller than the empty space left in the bin. This procedure is repeated until the end of the queue is reached. An item in the bin is served at speed 1. As we shall see, the probabilistic description of this model is not easy to handle; it involves an infinite-dimensional vector space (a space of strings).

The problems investigated in this paper concern the stability properties of this bandwidth allocation problem: Under some probabilistic assumptions on the sizes of the items, what is the maximum input rate under which the size of the queue converges in distribution?

Related models. A similar problem has been analyzed by Kipnis and Robert [21] with the FIFO algorithm: An item enters in the bin only if all the items arrived before it have left the queue. In particular an item in the queue cannot access the bin if it is not the first item in the queue. The stability problem is simpler in this case: the vector of the sizes of the items in the bin and the size of the first item in the queue is a Markov process. The lengths of the items in the queue, the first one excepted, are i.i.d. random variables with distribution $\mu$. To study the maximal throughput of this model, it is sufficient to calculate the output of the bin when the queue is saturated, that is, when it contains an infinite number of items. For the First Fit algorithm the situation is quite different. Since the queue is scanned to accommodate items in the bin, the sizes of the items in the queue are unlikely to remain independent and with the same initial distribution $\mu$. For example, there should be fewer small items at the beginning of the queue than at 
the end. Furthermore, if we saturate the queue, the output will not give the maximal output of the queue: If the size of the items are uniformly distributed on $[0,1]$, an infinite number of small items will be in the bin generating an infinite output.

Coffman and Stolyar [8] analyzed the stability of the algorithms First Fit and Best Fit when the services are constant equal to 1 . In this setting the problem is related to static bin packing problems. They prove that the natural condition $\lambda E\left(S_{1}\right)<C$ is sufficient for stability in the case of a symmetrical distribution of the sizes; in [7] the sufficiency for stability of the condition $\lambda E\left(S_{1}\right)<C$ is considered in a more complex communication network.

The First Fit algorithm with items having two possible sizes has been analyzed in [11]. In that paper the stability condition has been established and, more interesting, a curious transient behavior analyzed. The present paper is a continuation of this work. The case analyzed here requires a more detailed analysis of the evolution of the string structure than was necessary in [11].

Markov processes on strings occur also naturally in the multiclass queueing networks. Rybko and Stolyar [30] analyzed a two-node network with FIFO discipline. Due to clever arguments, the string structure is not really taken into account in their analysis. Bramson [2, 3] also analyzed the transience of a twonode network with a more complex interaction. In this case the stochastic process involves two interacting strings. Transience is proved via a very careful control of the process around a diverging path. Dumas [13] presented an analysis of some fluid equations for the string structure of Bramson's networks. Another set of FIFO networks, a generalization of Kelly's networks, has been analyzed in [4]. An interesting entropy function is used to study the convergence of fluid limits under the usual stability conditions. In this case, the FIFO discipline plays an important role in writing a set of fluid equations.

In the same vein, Gaurat, Malyshev, Men'shikov and Pelikh [18], Malyshev [25] and Serfozo [32] (and the references therein) investigated quite general models with strings but with a dynamic depending only on a finite number of components at the end (or the beginning) of the string. For the string valued processes we consider here, the dynamic depends, a priori, on all the components of the string since the queue is scanned as long as there is an empty space in the bin.

An overview. In this paper we give a necessary and sufficient condition under which the size of the queue converges in distribution (Theorems 14 and 15). If this condition has some interesting features, it is expressed as a quadratic functional of the input parameters; however, this is not the main point of the paper. The string valued Markov processes describing these models are in general difficult to analyze. The paper proposes an approach to analyze such processes. To keep the presentation simple, the simplest of these complicated models has been chosen. A companion paper [29] explores other aspects of these string processes.

To study the ergodicity properties of a finite-dimensional Markov process, a standard approach is the following: The behavior of the process is analyzed when 
the initial state is such that a subset $S$ of the coordinates is "large." When all the possible subsets $S$ have been considered, the ergodicity condition generally follows easily.

String valued processes can travel in infinitely many directions. To study the stability properties of these processes, one cannot recursively exhaust all the possibilities by inspection as is the case in a finite-dimensional setting. One of the conclusions of the paper is that it is better to consider the evolution of the distribution of the process at some random times rather than looking at the evolution of the states that the process visits at some random times as is usually the case. This is related, in some sense, to the case of continuous state space Markov chains: The recurrence of the chain is defined not in term of the number of visits to some specified states, but by the fact that, at some random times, the Markov chain has a specified distribution. Notice that although our framework is discrete (the state space is countable), these ideas are useful.

The framework of these Markov processes complicates technically the proofs of the results, even in some "simple" cases. See, for example, Section 4 where the ergodicity condition is quite intuitive, but its proof requires some discussion on the possible bifurcations of the system. This situation seems unavoidable, especially when the ergodicity condition is not natural at all (see Section 6).

The paper is organized as follows. We first prove that under some hypothesis, the Markov process describing the First Fit algorithm is ergodic if the "natural" condition is satisfied, that is, if the load of the system is less than 1 (see [11] for a discussion on this condition). In the other cases, the analysis is more intricate. The notion of smooth distribution on the state space is introduced. It is shown that at some random time the distribution of the process is smooth. Section 6 studies the fluid limits of the distributions of the process. The fluid limits can be described by piecewise linear processes in $\mathbb{R}_{+}^{2}$. The associated dynamical system turns out to be a product of random $2 \times 2$ matrices in $\mathbb{R}_{+}^{2}$; its stability properties are analyzed. These results are then used to derive the ergodicity and transience conditions for the Markov processes.

Our results concerning ergodicity use the formalism of fluid limits. The next section recalls some of the results in this domain.

2. Fluid limits. In this section $(X(t))$ is an irreducible Markov process on some countable space $\delta$ embedded in a normed space. We assume that the bounded subsets of $\&$ are finite. The rescaled process is defined by

$$
X_{x}(t)=\frac{\|X(t\|x\|)\|}{\|x\|},
$$

since $X(0)=x,\left\|X_{x}\right\|(0)=1$. The time variable and the space variable are scaled by a factor $\|x\|$. The following theorem is the combination of two results, one due to Filonov [17] and the other due to Rybko and Stolyar [30]; it gives an ergodicity criterion. 
THEOREM 1. If there exist an integrable stopping time $U$, constants $K$ and $\varepsilon>0$ such that

$$
\begin{gathered}
\limsup _{\|x\| \rightarrow+\infty} \frac{\mathbb{E}_{x}(\|X(U)\|)}{\|x\|} \leq 1-\varepsilon, \\
\limsup _{\|x\| \rightarrow+\infty} \frac{\mathbb{E}_{x}(U)}{\|x\|} \leq K,
\end{gathered}
$$

the Markov process $(X(t))$ is ergodic.

If the variable $X(t)$ has a second moment for all $t \geq 0$, for a fixed $K \geq 0$ sufficiently large, the hitting time

$$
H=\inf \{t \geq 0 \mid\|X(t)\| \leq K\},
$$

has a second moment of order $\|x\|^{2}$, that is,

$$
\limsup _{\|x\| \rightarrow+\infty} \frac{\mathbb{E}_{x}\left(H^{2}\right)}{\|x\|^{2}}<+\infty .
$$

Condition (2) requires that at some random time, $U /\|x\|$, the norm of the rescaled process $\left(X_{x}(t)\right)$ is, on average, below its initial value. This suggests the analysis of the sequences of processes $\left(X_{x}(t)\right)$, when $\|x\|$ tends to infinity. The limit of one of its converging subsequences is called a fluid limit. If one can prove that every fluid limit converges almost surely to 0 after some time $T$, then up to an integrability argument, Theorem 1 can be applied.

These scaling ideas are difficult to trace. The origin of this criterion is the Lyapounov stability test of ordinary differential equations (see [20] for the classical results). Has'minskii [19] seems to have been the first to use this test in a stochastic context to prove the stability of stochastic differential equations.

The discovery of some unexpected phenomena for the stability of queueing systems by Bramson [2, 3], Dumas [12], Lu and Kumar [24], Kumar and Seidman [22] and Rybko and Stolyar [30] among others, gave an impulse to the studies in this domain recently. Chen and Mandelbaum [5] used fluid limits to study Jackson networks. Dai [9] set a framework to apply these methods to prove Harris ergodicity for some queueing networks. Concerning transience criteria, Dai [10], Meyn [27] and Puhalskii and Rybko [28] obtained partial counterparts to the ergodicity results. In the context of diffusions, related ideas are used to prove the ergodicity of diffusions living in a domain with a boundary (see [14] and the references therein).

Relations (2) and (3) imply that one "controls" the process in space and time when it starts very far away from some fixed state. In a finite-dimensional context, one has to consider the process when some of the coordinates of the initial state are large. In general, Theorem 1 can then be applied when all the possibilities for the large coordinates have been considered. Applying Theorem 1 for our process turns 
out to be more difficult since the process can go to infinity in infinitely many ways. This is not strictly true as we shall see. We prove that the process may diverge only along some "patterns." We establish that, starting from any large arbitrary state, the process will eventually travel along some smooth random states. (This notion of smooth random state will be presented in detail later.)

3. The string valued Markov process. The items arrive according to a Poisson process $\mathcal{N}_{\lambda}$ with parameter $\lambda$; for $t \geq 0$, the quantity $\left.\left.\mathcal{N}_{\lambda}(] 0, t\right]\right)$ denotes the number of arrivals between 0 and $t$. The capacity of the bin $C$ is equal to 4 .

The sizes $\left(S_{i}\right)$ of the items form an i.i.d. sequence with a common distribution $F(d x)$ given by

$$
F(d x)=p \delta_{1}+q \delta_{2}+r \delta_{3},
$$

where $\delta_{x}$ is the Dirac measure in $x$ and $p, q, r$ are nonnegative numbers such that $p+q+r=1$. An item of size $s$ will also be called an item $s$.

The set of the possible sizes is denoted by $\mathcal{T}=\{1,2,3\}$ and $\mathcal{T}^{(\mathbb{N})}$ is the set of finite vectors with coordinates in $\mathcal{T}$; if $x \in \mathcal{T}^{(\mathbb{N})},\|x\|$ denotes the number of coordinates of $x$ and $\varnothing$ is the empty vector.

The sojourn times of the items in the bin is an i.i.d. sequence with an exponential distribution with parameter 1.

An element $X$ of the state space $\&$ of the Markov process describing the storage process can be written as $X=(B, L)$, where $L$ and $B$ are elements of $\mathcal{T}^{(\mathbb{N})}$, the set of finite vectors with coordinates in $\mathcal{T}$. The vector $B=\left(b_{j} ; j=1, \ldots,\|B\|\right)$ describes the sizes of the items in the bin, since these items fit in the bin,

$$
b_{1}+b_{2}+\cdots+b_{\|B\|} \leq C,
$$

and the vector $L=\left(l_{i} ; l=1, \ldots,\|L\|\right)$ represents the state of the queue, since the First Fit algorithm scans the queue from the beginning in search of an item that may fit in the bin. Any item in the queue cannot fit in the bin, that is, for any $i=1, \ldots,\|L\|$ the following inequality holds:

$$
l_{i}+b_{1}+b_{2}+\cdots+b_{\|B\|}>C .
$$

Since $C=4$, the possible values of $B$ are the following:

$$
\varnothing,(1),(1,1),(1,1,1),(1,1,1,1),(1,1,2),(1,2),(1,3),(2),(2,2),(3) .
$$

Notice that the order of the components in $B$ has no importance for the dynamic of the system; for this reason we shall consider $B$ as a set. The order is important for the vector $L$ since the First Fit discipline checks if the first coordinate $l_{1}$ fits in the bin, then the coordinates $l_{2}, l_{3}$, and so on. The vector $L$ is a string of $1,2,3$.

If $(X(t))=((B(t), L(t))$ is the state of the system at time $t,(X(t))$ is a Markov process with the following transitions: 
1. Arrival. At rate $\lambda$ an item of size $s$ arrives at the bin. If it does not fit in the bin, the element $s$ is concatenated at the end of the vector $L(t)$.

2. Departure. At rate 1, each item in the bin leaves the bin. In the case of a departure, the first element of the queue that fits, if any, is moved in the bin, and then the second, and so on.

It is not difficult to show that $(X(t))$ is an irreducible Markov process on 8 . We shall say that the model is stable when $(X(t))$ is an ergodic Markov process on $\&$. In [11] it has been proved that the condition $\lambda \mathbb{E}\left(S_{1}\right) \leq C$ is necessary for the stability of the system; that is, the Markov process $(X(t))$ is transient if $\lambda \mathbb{E}\left(S_{1}\right)>C$.

Definition 2. The norm $\|X\|$ of the state $X=(B, L) \in \delta$ is the sum of the $\|B\|$ and $\|L\|$. The load $W(X(t))$ of $(X(t))=(B(t), L(t))=\left(\left(b_{i}(t)\right),\left(l_{j}(t)\right)\right)$ is defined as

$$
W(X(t))=\sum_{i=1}^{\|B(t)\|} b_{i} \sigma_{i}^{0}(t)+\sum_{j=1}^{\|L(t)\|} l_{j} \sigma_{j}(t),
$$

where, for $i \in\{1, \ldots,\|B\|\}$ and $j \in\{1, \ldots,\|L\|\}, \sigma_{i}^{0}(t)$ is the residual service time of the item $b_{i}(t)$ and $\left(\sigma_{j}(t)\right)$ the service time of the item $l_{j}(t)$.

Notice that the load of the system increases at rate $\lambda \mathbb{E}\left(S_{1}\right)$ in average and decreases rate 4 at most.

4. When the natural condition is sufficient for ergodicity. We study a case where it is not necessary to know much about the structure of the $L$-component of the initial state. The following lemma gives an estimation of the wasted space when there are only two possible sizes: 1 and 2 .

LEMMA 3. Under the conditions $\lambda \mathbb{E}\left(S_{1}\right)<4$, if $r=0$ (only items 1 and 2 arrive) and

$$
\tau=\inf \{t \geq 0 \mid\|L(t)\|=0\} \quad \text { and } \quad D=\int_{0}^{\tau} 1_{\left\{b_{1}(t)+\cdots+b_{\|B(t)\|}(t)<4\right\}} d t,
$$

then there exist some constants $K_{1}$ and $K_{2}$ such that

$$
\mathbb{E}_{x}(D) \leq K_{1} \log (1+\|x\|)+K_{2}
$$

for any $x=(l, b) \in \S$.

PROOF. The variable $D$ is the duration of time during which the bin is not full during a busy period. Notice first that there is no waste of space as long as there are items 1 in the $L$-component of $(X(t))$. We can therefore assume that $l$ is a string of items 2 . In this context the only possibility to waste space with a nonempty queue 
is when the state $(B(t))$ of the bin is $(1,1,1)$ or $(2,1)$. We set $A_{0}=l$ and $T_{0}=0$ and by induction we define

$$
\begin{aligned}
& T_{n+1}=\inf \left\{t>T_{n}: C(t-)=4, C(t)<4,\right. \\
& \text { and all the items } \left.2 \text { present at time } T_{n} \text { are served at time } t\right\}
\end{aligned}
$$

with $C(s)=b_{1}(s)+\cdots+b_{\|B(s)\|}(s)$ for $s \geq 0$ and $A_{n}=\left\|L\left(T_{n}\right)\right\|$ for $n \geq 1$. Notice that $L\left(T_{n}\right)$ is necessarily a (possibly empty) string of items 2 . The sequence $\left(B\left(T_{n}\right), A_{n}\right)$ is clearly a Markov chain.

If $b$, the initial state of the bin, is $(1,1,1)$ : As long as there is at least an item 1 in the queue, because of the First Fit discipline, items 2 are ignored. Since $\lambda p \leq \lambda \mathbb{E}\left(S_{1}\right)<4$, after an integrable amount of time not depending on $\|l\|$, at least two places will be vacant in the bin and consequently an item 2 will enter the bin. In this situation the number of items 1 is the number of customers of an $M / M / 4$ queue with parameter $\lambda p$ for the input rate and 1 for the service rate.

If $b=(2,1)$, we have two cases to discuss.

1. $\lambda p<2$. This condition clearly implies that, with probability 1 , at some time there will no item 1 in the system and, consequently, a second item 2 will enter the bin. The expected value of this duration of time is easily seen to be bounded with respect to $\|l\|$. Starting from that time, only items 2 are served as long as the initial items 2 are present (since these items are located at the beginning of the queue, the First Fit algorithm selects them). When the initial items 2 have been served, the queue is an i.i.d. string of items 1 and 2 . With probability 1 , at least two items 1 enter in the bin. Later, when the number of items 1 in the system is 1 , the system will waste some space; this is precisely the definition of time $T_{1} . A_{1}$ is the number of items at that time.

2. $\lambda p>2$. This condition implies that, if the state of the bin does not change, the arriving items 1 will saturate two places in the bin. In this case, the number of items 1 is the number of customers of a transient $M / M / 2$ queue starting with one customer (in the bin at time 0 ). A change in the state of the bin may occur only if this transient queue is empty.

(a) The $M / M / 2$ queue never reaches the empty state. After some small amount of time (i.e., its expected value is bounded with respect to $\|l\|$ ), the bin will be full with an item 2 and two items 1 . The condition $\lambda \mathbb{E}\left(S_{1}\right)<4$ implies that $\lambda q<1$; therefore, with probability 1 after some period of time the system will not contain any items 2 . At that moment the state of the bin will be $(1,1,1,1)$. [Recall that $\lambda p \leq \lambda \mathbb{E}\left(S_{1}\right)<4$.] It is easily seen that, with probability 1 , the total number of items 1 will be less than 2 . An item 2 will be in the bin at that time; this is the starting situation.

(b) The queue reaches the empty state. Two items 2 occupy the bin. The initial items 2 are served. In this situation, $T_{1}$ is the next time there is some wasted space. 
Notice that the case (a) occurs only a geometrically distributed number of times.

Hence, the duration of time between time 0 and $T_{1}$ when the bin is not full has a bounded expected value (with respect to $\|l\|$ ).

Using Proposition 16 of the Appendix, it is easy to check that there exists some constant $c>0$ such that the following convergence holds in $L_{1}$ and almost surely,

$$
\lim _{\|x\| \rightarrow+\infty} \frac{\mathbb{E}_{x}\left(T_{1}\right)}{\|x\|}=c .
$$

(Its calculation is possible but not interesting for our purpose.) If $I$ is the duration of time between 0 and $T_{1}$ when the bin is not full, the expected value of the load at time $T_{1}$ satisfies the following inequality (all the service times are i.i.d. exponentially distributed random variables with parameter 1):

$$
\mathbb{E}_{x}\left(W\left(X\left(T_{1}\right)\right)\right) \leq \mathbb{E}_{x}(W(x))+\mathbb{E}\left(\sum_{i=1}^{\mathcal{N}_{\lambda}\left(\left[0, T_{1}\right]\right)} S_{i}\right)-4 \mathbb{E}_{x}\left(T_{1}-I\right) .
$$

Using Wald's formula ( $T_{1}$ is a stopping time), we get

$$
\mathbb{E}_{x}\left(W\left(X\left(T_{1}\right)\right)\right) \leq \mathbb{E}_{x}(W(x))+\left(\lambda \mathbb{E}\left(S_{1}\right)-4\right) \mathbb{E}_{x}\left(T_{1}\right)+4 \mathbb{E}_{x}(I) .
$$

Since there are no items 1 in the queue at 0 and $T_{1}$, we have $\mathbb{E}_{x}(W(X(0)))=2\|x\|$ and $\mathbb{E}_{x}\left(W\left(X\left(T_{1}\right)\right)\right)=3+2 \mathbb{E}_{x}\left(A_{1}\right)$. The quantity $\mathbb{E}_{x}(I)$ being bounded with respect to $\|x\|$, it follows that

$$
2 \limsup _{\|x\| \rightarrow+\infty} \frac{\mathbb{E}_{x}\left(A_{1}\right)}{\|x\|}=\limsup _{\|x\| \rightarrow+\infty} \frac{\mathbb{E}_{x}\left(W\left(X\left(T_{1}\right)\right)\right)}{\|x\|} \leq 2+c\left(\lambda \mathbb{E}\left(S_{1}\right)-4\right),
$$

where $W(\cdot)$ is the load (see Definition 2). Consequently, there exist $a_{0}$ and $\alpha<1$ such that for $\|x\|>a_{0}$,

$$
\begin{gathered}
\mathbb{E}_{x}\left(A_{1}\right) \leq \alpha\|x\|, \\
\gamma=-\log \left(\frac{1+\alpha\|x\|}{1+\|x\|}\right)>0 .
\end{gathered}
$$

If we set

$$
v=\inf \left\{n \geq 1 \mid A_{n} \leq a_{0}\right\}
$$

the sequence

$$
\left(Z_{n}\right)=\left(\log \left(1+A_{n \wedge \nu}\right)+\gamma(n \wedge v)\right)
$$

is a supermartingale. Indeed, if $\left(\mathcal{F}_{n}\right)$ is the natural filtration associated to the sequence $\left(A_{n}\right)$, on the event $\{v>n\}$ the Markov property gives the equality

$$
\begin{aligned}
\mathbb{E}\left(Z_{n+1} \mid \mathcal{F}_{n}\right)-Z_{n} & =\mathbb{E}_{\left(B\left(T_{n}\right), A_{n}\right)}\left(\log \left(1+A_{1}\right)\right)-\log \left(1+A_{n}\right)+\gamma \\
& \leq \log \left(1+\mathbb{E}\left(A_{1} \mid A_{n}\right)\right)-\log \left(1+A_{n}\right)+\gamma \leq 0,
\end{aligned}
$$


by Jensen's inequality and relations (5) and (6). Consequently, $\mathbb{E}\left(Z_{n}\right) \leq Z_{0}$, hence $\gamma \mathbb{E}(n \wedge v) \leq \mathbb{E}\left(Z_{n}\right) \leq Z_{0}$. By letting $n$ go to infinity, we get

$$
\mathbb{E}_{x}(\nu) \leq \frac{\log (1+\|x\|)}{\gamma} .
$$

For $n \geq 1$, the bin is always full between $T_{n}$ and $T_{n+1}$, except during some integrable period whose expected value is bounded with respect to the size of the initial state. By Wald's formula, the contribution of the $v$ cycles in the integral defining $D$ is bounded by $K \mathbb{E}_{x}(\nu) \leq K \log (1+\|x\|) / \gamma$, for some constant $K$.

Since $\lambda \mathbb{E}\left(S_{1}\right)<1$, Proposition 6 of [11] shows that the system is ergodic. Consequently, starting from the state $\left.\left(B\left(T_{\nu}\right), A_{\nu}\right)\left(\leq a_{0}\right)\right)$, the hitting time of the empty state $\varnothing$ is integrable and with an expected value bounded with respect to $\|x\|$. Therefore, the expected value of the contribution of this period in the integral defining $D$ is bounded with respect to $\|x\|$. The lemma is proved.

Now we consider the general case with three sizes. The condition $\lambda \mathbb{E}\left(S_{1}\right)<4$ turns out to be sufficient for ergodicity when $\lambda p>1$.

PROPOSITION 4. If $\lambda \mathbb{E}\left(S_{1}\right)<4$ and $\lambda p>1$, then $(X(t))$ is an ergodic Markov process.

ProOF. Let $\left(x_{n}\right)=\left(b_{n}, l_{n}\right)$ a sequence of $s$ whose norm converges to infinity. Since the number of configurations in the bin is finite, by taking subsequences we can suppose that the sequence of the initial states in the bin $\left(b_{n}\right)$ is constant, hence $\left(x_{n}\right)=\left(b, l_{n}\right)$. Using Proposition 5 of [11], we can assume that for the states $\left(x_{n}\right)$ the bin is not full. Consequently, $\left(l_{n}\right)$ does not contain any items 1 ; it is a sequence of strings of items 2 and 3 .

We denote by $\tau$ the first time when the bin is not full after all the initial items 2 and 3 have left the system; $\tau$ is clearly a (possibly infinite) stopping time. If $D$ is the duration of time between time 0 and $\tau$ during which the bin is not full, we claim that $D$ is integrable and, moreover,

$$
\lim _{n \rightarrow+\infty} \frac{\mathbb{E}_{x_{n}}(D)}{\left\|x_{n}\right\|}=0
$$

If our assertion is true, between 0 and $\tau$ the load of the system is decreased at rate 4 , except during some periods of total duration $D$; that is, for $t \geq 0$ we have

$$
\sum_{i=1}^{\|b\|} b_{i} \sigma_{i}^{0}+\sum_{i=\|b\|+1}^{\left\|l_{n}\right\|+\|b\|} l_{n, i} \sigma_{i}^{0}+\sum_{i=1}^{\left.\left.\mathcal{N}_{\lambda}(] 0, t \wedge \tau\right]\right)} S_{i} \sigma_{i}-4(t \wedge \tau-D) \geq 0 .
$$

The sequences $\left(\sigma_{i}\right)$ and $\left(\sigma_{i}^{0}\right)$ are the respective service times of the arriving items and of the initial items. These variables are independent and exponentially 
distributed with parameter 1 . Taking the expectation of the two members of this inequality, we get the relation

$$
\mathbb{E}_{x_{n}}(t \wedge \tau)\left(4-\lambda \mathbb{E}\left(S_{1}\right)\right) \leq\left\|x_{n}\right\|+4 \mathbb{E}_{x_{n}}(D) .
$$

By letting $t$ go to infinity, according to our assumption on $\left(\mathbb{E}_{x_{n}}(D)\right)$ we obtain the inequality,

$$
\limsup _{n \rightarrow+\infty} \frac{\mathbb{E}_{x_{n}}(\tau)}{\left\|x_{n}\right\|} \leq \frac{1}{4-\lambda \mathbb{E}\left(S_{1}\right)} .
$$

In the same manner, the following inequality holds:

$$
\mathbb{E}_{x_{n}}(W(X(t \wedge \tau))) \leq W\left(x_{n}\right)+\left(\lambda \mathbb{E}\left(S_{1}\right)-4\right) \mathbb{E}_{x_{n}}(\tau \wedge t)+4 \mathbb{E}_{x_{n}}(D) .
$$

Fatou's lemma and Lebesgue's theorem give when $t$ goes to infinity,

$$
\mathbb{E}_{x_{n}}(W(X(\tau))) \leq W\left(x_{n}\right)+\left(\lambda \mathbb{E}\left(S_{1}\right)-4\right) \mathbb{E}_{x_{n}}(\tau)+4 \mathbb{E}_{x_{n}}(D) .
$$

Since all the initial items 2 and 3 are served at time $\tau$, at most two of the initial items can be served at the same time; hence,

$$
\mathbb{E}_{x_{n}}(\tau) \geq \frac{\left\|x_{n}\right\|}{2} \geq \frac{W\left(x_{n}\right)}{6}
$$

consequently,

$$
\limsup _{n \rightarrow+\infty} \frac{\mathbb{E}_{x_{n}}(W(X(\tau)))}{W\left(x_{n}\right)} \leq 1+\frac{\lambda \mathbb{E}\left(S_{1}\right)-4}{6}<1 .
$$

By using the fact that $W(\cdot)$ is an equivalent norm to $\|\cdot\|$ on $\delta$, relations (8) and (9) and Theorem 1 show that the Markov process $(X(t))$ is ergodic.

All we have to prove now is that $\left(\mathbb{E}_{x_{n}}(D)\right)$ is negligible with respect to $\left\|x_{n}\right\|$ when $n$ is large. There are several possibilities for $b$, the common content of the bin for the initial states $\left(x_{n}\right)$. We discuss the different cases. Throughout this discussion, we shall say that random variable $H$ is a "bounded integrable variable" if the sequence $\left(\mathbb{E}_{x_{n}}(H)\right)$ is bounded with respect to $\|x\|$.

1. If $b$ is $(1,1,1)$ or $(1,1,1,1)$. As long as there is at least an item 1 in the queue, all the other items are ignored. The condition $\lambda \mathbb{E}\left(S_{1}\right)<4$ implies that $\lambda p<4$. From the point of view of the items 1 , the system is a stable $M / M / 4$ queue. Hence the first time there will be at least two empty places is a bounded integrable variable. At that time, an item 2 will be inserted in the bin. Notice that for this period, the duration of time during which the bin is not full is a bounded integrable variable.

2. If $b$ has at least an item 2. The items 3 are not taken into account as long as the bin contains at least an item 2 or two items 1 . In this case the items 3 are ignored. Consequently, a string of the initial items 3 builds up at the beginning of the queue. Since the condition $\lambda \mathbb{E}\left(S_{1}\right)<4$ implies

$$
\lambda p+2 q<4,
$$


the system with the items 1 and 2 is stable (see [11]). Lemma 3 shows that until an item 3 enters the bin the wasted space is negligible compared to the number of initial items 2 .

3. If $b$ contains a 3 . Clearly one can assume that $l_{n}$ is a string of items 3 . Otherwise, if at some moment 2 enters in the bin, the cases considered above show that the 1 and 2 will be cleared from the system until an item 3 is in the bin. The condition $\lambda p>1$ implies that the residual space in the bin left by the items 3 is saturated by the items 1 . Consequently, the duration of time the bin is not full is a bounded integrable variable.

This discussion shows that the assertion is proved and consequently, the proposition.

The result of the above proposition is fairly easy to understand: Under the condition $\lambda p>1$ basically there is no waste of space so that the natural condition $\lambda \mathbb{E}\left(S_{1}\right)<4$ is sufficient for the ergodicity of $(X(t))$. Notice however that the proof of this intuitive result (Lemma 3 and Proposition 4 ) has required the detailed analysis of the possible evolutions starting from a given initial state. As we shall see, the situation is more delicate in the case $\lambda p<1$.

5. Smoothing the initial state. This section studies the properties of the dynamic on the string structure of the queue (the $L$-component of the Markov process). From now on, we shall assume that $\lambda p<1$ and that the initial states are strings of items 2 and 3 (see Proposition 5 of [11]). Even if the $L$-component of the initial states have only items 2 and 3 , they are still too complicated to use fluid limits starting from these states. Loosely speaking, the main idea is the following: after some time the initial disorder of the $L$-component is smoothed; that is, it will have some regularity properties. If the initial state is a "smooth random" state, a fluid analysis of the Markov process is then possible. This will be done in the next section. Compared to our previous analysis [11], this smoothing procedure is an important additional step. It is discussed in a more general context in [29].

Definition 5. For $X(0)=x \in \delta$ and $t \geq 0$, if $X(t)=(B(t), L(t))$ and $L(t)=\left(l_{i}(t)\right)$,

$$
\begin{aligned}
& v_{x, 1}(t)=\inf \left\{k \geq 1: l_{k}(t)=1\right\}, \\
& v_{x, 2}(t)=\inf \left\{1 \leq k<v_{x, 1}(t): l_{k}(t)=2\right\}, \\
& v_{x, 3}(t)=\inf \left\{1 \leq k \leq v_{x, 2}(t): l_{k}(t)=3\right\},
\end{aligned}
$$

with the convention $\inf \varnothing=+\infty$. If the initial state is without ambiguity, the subscript $x$ is omitted; in the same way, the notation $v_{a}$ is used for $v_{a}(0)$.

The next definition formalizes the notion of a "smooth random" state, in fact, the notion of a smooth distribution on $\$$. 
Definition 6. For integers $l, m, n$ we define the distribution $R_{l, m, n}(d x)$ on $\mathcal{T}^{(\mathbb{N})}$ by

$$
R_{l, m, n}(d x)=\delta_{3}(d u)^{(l)} \otimes F_{2,3}(d u)^{(m)} \otimes F(d u)^{(n)},
$$

where $G(d x)^{(n)}$ is the $n$th power of the distribution $G(d x)$ and $F_{2,3}(d x)$ is the conditional distribution $F_{2,3}(d x)=\left(q \delta_{2}+r \delta_{3}\right) /(q+r)$.

A distribution $\mu$ on $\delta$ is smooth if its $L$-component is in the convex hull of the $R_{l, m, n}, l, m, n \in \mathbb{N}$, that is, if there exists a probability distribution $\left(q_{i}\right)$ on $\mathbb{N}^{3}$ such that

$$
\mu(L \in d x)=\sum_{i \in \mathbb{N}^{3}} q_{i} R_{i}(d x)
$$

The distribution $R_{l, m, n}$ is the distribution of the concatenation of several i.i.d. strings. The $L$-component of a distribution of type $R_{0,0, n}(d x)$ is just an i.i.d. string of length $n$ with distribution $F$.

PROPOSITION 7. If $\lambda \mathbb{E}\left(S_{1}\right)<4$, for any stopping time $U$ greater than the first time when all the initial items have left the queue, the distribution of $X(U)$ is smooth.

Proof. We denote by $M(t)$, the number of initial items in the queue at time $t$. A tag is inserted after the last initial item in the queue; $M(t)$ is in fact the position of the tag at time $t ;(M(t))$ remains constant equal to 0 after it has reached 0 . We first give a rough picture of the evolution of the queue. After time 0 , the new items arrive behind the tag at rate $\lambda$. Recall that the queue of our initial state has no item 1. As long as some initial items 2 are in the queue, the First Fit algorithm picks (possibly) only items 1 after the tag. The departure of some of the items 1 builds a string of 2's and 3's after the tag. In the case where all the initial items 2 are processed and some initial items 3 remain, the next items 2 are picked after the tag. In this case, a string of items 3 will build up behind the tag and before the string of 2's and 3's.

The notation $\tilde{v}_{a}, a \in \mathcal{T}$ is analogous to Definition 5 except that it concerns only the portion of the queue after the tag,

$$
\begin{aligned}
& \tilde{v}_{1}(t)=\inf \left\{k>M(t): l_{k}(t)=1\right\}, \\
& \tilde{v}_{2}(t)=\inf \left\{M(t)<k<\tilde{v}_{x, 1}(t): l_{k}(t)=2\right\}, \\
& \tilde{v}_{3}(t)=\inf \left\{M(t)<k \leq \tilde{v}_{x, 2}(t): l_{k}(t)=3\right\} .
\end{aligned}
$$

Notice that if $\tilde{v}_{3}(t)$ is finite, then necessarily $\tilde{v}_{3}(t)=M(t)+1$. The variable $\tilde{L}(t)$ is the substring at the end of the queue consisting of the items located after the tag, $\tilde{L}(t)$ is the string $L(t)$ shifted $M(t)$ times. Consequently, if $U \leq t$, then $\tilde{L}(t)=L(t)$ and $\tilde{v}_{a}=v_{a}$ for $a \in \mathcal{T}$. 
ASSERTION. If $\tau$ is a stopping time, then conditionally on $\tilde{v}_{a}(\tau), a \in \mathcal{T}$, and $\|L(\tau)\|$, the distribution of $\tilde{L}(\tau)$ is given by (10) for some convenient $l, m$ and $n \in \mathbb{N}$.

Since $L(U)=\tilde{L}(U)$ (the initial items are served at time $U$ ), the proposition will be then proved if the assertion is.

To show this claim, we shall assume that all the $\tilde{v}_{a}(\tau), a=1,2,3$, are finite. The analysis for the other cases is analogous. The string $\tilde{L}(\tau)$ is thus the concatenation of three strings $\tilde{L}(\tau)=\left(H_{3}, H_{2}, H_{1}\right)$, with

$$
\begin{array}{llrl}
H_{3} & =(3, \ldots, 3), & \left\|H_{3}\right\| & =\tilde{v}_{2}(\tau)-1 ; \\
H_{2} & =\left(2, l_{\tilde{v}_{2}(\tau)+1}, \ldots, l_{\tilde{v}_{1}(\tau)-1}\right), & & \left\|H_{2}\right\|=\tilde{v}_{1}(\tau)-\tilde{v}_{2}(\tau) ; \\
H_{1} & =\left(1, l_{\tilde{v}_{1}(\tau)+1}, \ldots, l_{|L(\tau)|}\right), & \left\|H_{1}\right\| & =\|L(\tau)\|-\tilde{v}_{1}(\tau) .
\end{array}
$$

For the rest of the proof, all the probabilistic statements are supposed to be conditioned by the values of the $\tilde{v}_{a}(\tau)$ and $\|L(t)\|$. Between time 0 and $\tau$ the First Fit algorithm never scanned the queue after the position $\tilde{v}_{1}(\tau)$, otherwise the item 1 located there would have been taken in the bin. The string $H_{1}$ is thus independent of $\mathrm{H}_{3}$ and $\mathrm{H}_{2}$. The first item 1 of $H_{1}$ is followed by the $\left(\|L(\tau)\|-\tilde{v}_{1}(\tau)-1\right)^{+}$items which arrived after that 1 ; hence it is an i.i.d. sequence with distribution $F(d u)$.

In the same way for the string $H_{2}$, the First Fit algorithm never scanned the queue in search of a 2 after the position $\tilde{v}_{1}(\tau)$. Consequently, the string $H_{2}$ consists of all the items arrived between the items located at the positions $\tilde{\nu}_{2}(\tau)$ and $\tilde{v}_{1}(\tau)$, with all the items 1 removed. The first item 2 in $H_{2}$ is followed by an i.i.d. string of length $\left(\tilde{v}_{1}(\tau)-\tilde{v}_{2}(\tau)-1\right)^{+}$and distribution $F(d u \mid u \geq 2)$. The assertion is proved.

PROPOSITION 8. If $\lambda \mathbb{E}\left(S_{1}\right)<4, \lambda p<1$ and $U_{0}$ is the first time $t$ after all the initial items have left the queue that $B(t)=(2,1)$, then

$$
\sup _{x \in s_{1}} \mathbb{E}_{x}\left(\left(\frac{U_{0}}{\|x\|}\right)^{2}\right)<+\infty \text { and } \sup _{x \in s_{1}} \mathbb{E}_{x}\left(\left(\frac{\left\|X\left(U_{0}\right)\right\|}{\|x\|}\right)^{2}\right)<+\infty,
$$

where $\$_{1}$ is the subset of the states of $\&$ for which the bin is not full,

$$
s_{1}=\left\{x=(b, l) \in s: b_{1}+\cdots+b_{\|b\|}<4\right\} .
$$

Proof. The initial state $X(0)$ is given by $x=(B, L)$ with $L=\left(l_{1}, \ldots, l_{p}\right)$ for some $p \geq 1$. We denote by $T_{2}$ (resp. $T_{3}$ ) the time when all the initial items 2 (resp. 3) have left the queue. The variable $T$ is the first time when all the initial items have left the queue; $T$ is clearly stopping time bounded by $T_{2}+T_{3}$.

For a fixed $k \in\{1, \ldots, p-1\}$, we define $\check{x}=(B, \breve{L})$ where $\breve{L}$ is the same string as $L$ except the components $k$ and $k+1$ are permuted. For $1 \leq i<p$, the quantities 
$\tau_{i}, \check{\tau}_{i}$ denote, respectively, the waiting time necessary for the $i$ th item $l_{i}$ to enter the bin when the initial state is, respectively, $x, \check{x}$. We assume that for these two initial states, the arrival stream and the services associated with the items are the same. There are two cases:

1. If $\tau_{k+1}<\tau_{k}$, in both systems the item $l_{k}$ will enter the bin at time $\tau_{k}$, thus $\check{\tau}_{k}=\tau_{k}$.

2. Otherwise, when $\tau_{k+1} \geq \tau_{k}$ and the initial state is $\check{x}$, at time $\tau_{k}$ the First Fit algorithm checks if the item $l_{k+1}$ fits in the bin and after the item $l_{k}$ is checked.

Hence, in any case $\tau_{k} \leq \check{\tau}_{k}$. By induction, the quantity $\mathbb{E}_{x}\left(T_{2}\right)$ is thus bounded by $\mathbb{E}_{x^{\prime}}\left(T_{2}\right)$ where $x^{\prime}=\left(B, L^{\prime}\right)$ is the initial state given by $L^{\prime}=(3, \ldots, 3,2, \ldots, 2)$, $L^{\prime}$ is a permutation of $L$ with all the items 3 at the beginning. Similarly, the relation $\mathbb{E}_{x}\left(T_{2}\right) \leq \mathbb{E}_{x^{\prime \prime}}\left(T_{2}\right)$ holds if $T_{2}$ is the time to get rid of the initial 2's and $x^{\prime \prime}=\left(B, L^{\prime \prime}\right)$, where $L^{\prime \prime}$ is a permutation of the $L$-component of $x$ when all the items 3 are at the head of the queue. To bound $\mathbb{E}_{x}\left(T^{2}\right)$ it is sufficient to give an upper bound for $\mathbb{E}_{x^{\prime \prime}}\left(T_{2}^{2}\right)$ and $\mathbb{E}_{x^{\prime}}\left(T_{3}^{2}\right)$.

1. Items 2 are at the beginning. We can assume that the bin does not contain a 3 at time 0 (otherwise, as soon as it leaves it is replaced by an item 2). As long as an item 2 is at the head of the queue, the system works only with items of size 1 and 2 . When the system without items 3 has at most one item 1 in the bin and an item 3 enters in the bin, then all the initial items are served consecutively. The estimation of $T_{3}$ is thus reduced to estimation of the time to empty the system without items 3 . Since the condition $\lambda \mathbb{E}\left(S_{1}\right)<4$ implies that $\lambda(p+2 q) /(p+q)<4$, the system without items 3 is ergodic. Using the ergodicity result of [11] and inequality (4) of Theorem 1, we get that $\mathbb{E}_{x^{\prime}}\left(T_{3}^{2}\right) \leq A_{1}\|x\|^{2}$ (notice that $\left\|x^{\prime}\right\|=\|x\|$ ), for some constant $A_{1}$.

2. Items 3 are at the beginning:

(A) If the initial state of the bin has an item 3, all the initial 3's are served consecutively and then the initial 2's are served. For a convenient constant $A_{2}$, one easily gets that $\mathbb{E}_{x^{\prime \prime}}\left(T_{2}^{2}\right) \leq A_{2}\|x\|^{2}$.

(B) If there is a 2 in the bin and at least a 1, the situation is more interesting. At the difference of the previous case, an item 3 can enter the bin before some of the initial items of size 2 if at some moment the state of the bin is $(2,1)$ (there is an empty place of size 1) and that no new item 1 arrives before a departure form the bin. If the item 2 leaves before the item 1 then the item 3 at the head of the queue enters the bin, and then all the other initial items 3 ; otherwise if the item 1 leaves first, an additional 2 enters the bin, then all the initial items 2 are processed. Since $\lambda p<1$, if there are sufficiently many 2 's in the queue, one of this two cases will occur with probability 1 (if it is not the case, the 3's occupy the bin and it is finished). We thus get a constant $A_{3}$ such that $\mathbb{E}_{x^{\prime \prime}}\left(T_{2}\right) \leq A_{3}\|x\|$. 
At time $T$ all the initial items have left the queue. Since

$$
\left.\left.X(T) \leq\|x\|+\mathcal{N}_{\lambda}(] 0, T\right]\right),
$$

Wald's formula and the above estimation show that $\|X(T)\|$ is bounded by a constant times $\|x\|$.

Now we have to estimate $\bar{T}$ the first time when the state of the bin is $(2,1)$. It is sufficient to prove that, if the initial state is $x, \bar{T}$ has a second moment of the order $\|x\|^{2}$. The first step is to get rid of items 3 . If there is one in the bin and if some of them are located at the head of the queue and one has to process these ones until an additional item 1 or 2 enters the bin, there are two possibilities:

(a) The bin has at least 1 item 1 . Since $\lambda p<1$, after some time the queue will not have any item 1 and the bin will have two items 1 .

(a1) If, at that time, there are sufficiently many items 2 in the queue, the state of the bin will reach the state $(2,1)$ with probability 1 .

(a2) If not. All the items 3 in the queue at that time are served. When this is finished, the condition $\lambda p<1$ implies that the number of items 1 is tight (as a family of random variables indexed by $x$, the initial state). The items 2 accumulated during that time are served, consequently with probability 1 , the state of the bin will be $(2,1)$.

(b) The state of the bin is $(2,2)$ and the initial items 2 are served at rate 2 . When this is finished, with probability 1 , two items 1 enters in the bin. This is the situation of the previous case.

It is easily seen that each of the steps we have described has a duration with a second moment of the order $\|x\|^{2}$. The proposition is proved. The last inequality is a consequence of Wald's formula applied to the stopping time $U_{0}$.

6. A random dynamical system in $\mathbb{R}_{+}^{2}$. In this section we assume that $\left(\mu_{n}\right)$ is a sequence of smooth distributions on 8 (see Definition 6) such that

$$
\mu_{n}(B=(2,1), L \in d x)=\mathbb{E}\left(R_{a_{n}, b_{n}, 0}(d x)\right) ;
$$

that is, if $f$ is a non-negative measurable function on $\mathcal{T}^{(\mathbb{N})}$,

$$
\mathbb{E}_{\mu_{n}}\left(f(L(0)) 1_{\{B(0)=(2,1)\}}\right)=\mathbb{E}\left(\int_{\mathcal{T}^{(\mathbb{N})}} f(x) R_{a_{n}, b_{n}, 0}(d x)\right),
$$

where $a_{n}$ and $b_{n}$ are random variables such that the convergence

$$
\lim _{n \rightarrow+\infty} \frac{a_{n}}{n}=a \text { and } \quad \lim _{n \rightarrow+\infty} \frac{b_{n}}{n}=b
$$

holds in $L_{1}$. We assume that $a$ and $b$ are nonnegative integrable random variables and $\mathbb{P}(a+b>0)=1$. The $B$-component of $\mu_{n}$ is $(2,1)$ and the $L$-component of the distribution $\mu_{n}$ does not have an item 1 in the queue; it is the concatenation of $a_{n}$ items 3 followed by an i.i.d. string of length $b_{n}$ of 2's and 3's with respective probability $q /(q+r)$ and $r /(q+r)$. 
DEFINITION 9. A sequence $\left(X_{n}\right)$ of random variables is equivalent to $\left(\alpha_{n}\right)$ if the sequence $\left(X_{n} / \alpha_{n}\right)$ converges to 1 in $L_{1}(\mathbb{P})$.

A random transition of the fluid model. If the initial distribution is given by $\mu_{n}$, the initial state of the bin is $(2,1)$. If there is a departure before a new arrival:

1. With probability $1 / 2$, this is item 2 and then an item 3 enters the bin and then all the other $a_{n}-1$ items 3 .

2. With probability $1 / 2$ this is item 1 , another item 2 enters the bin, and then all the other initial items 2 will be served consecutively.

We remark that the dynamic of the system is influenced by the fact that either the 2 leaves first or not. This is also true at the fluid level as we shall see. A similar phenomenon has been already encountered in the model analyzed in [11]. Here the randomness remains because of this $1 / 2-1 / 2$ transition and not because there are many possibilities for the content of the bin. In [29], it is shown that this random bifurcation may depend on the current state; this is not the case here.

If the distribution of $X(0)$ is given by $\mu_{n}$, then $\|X(0)\|$ is equivalent to $((a+b) n)$. The next proposition shows that, up to a linear transformation, the distribution of $X$ at a stopping time has a property similar to identity (12).

PROPOSITION 10. If $U_{1}$ is the first time when all the initial items 2 have left the queue, the initial items 2 in the bin have been served and the state of the bin is $(2,1)$, there exist $\mathcal{F}_{U_{1}}$-measurable random variables $A_{n}, B_{n}$ such that the following relation holds:

$$
\mathbb{P}_{\mu_{n}}\left(B\left(U_{1}\right)=(2,1), L\left(U_{1}\right) \in d x\right)=\mathbb{E}_{\mu_{n}}\left(R_{A_{n}, B_{n}, 0}(d x)\right),
$$

and a random matrix $M$ such that the convergence

$$
\lim _{n \rightarrow+\infty} \frac{1}{n}\left(A_{n}, B_{n}\right)=M \cdot(a, b)
$$

is true almost surely and in $L_{1}$. The random matrix $M$ has two possible values with equal probability,

$$
m_{1}=\left(\begin{array}{cc}
1 & \frac{1-p-q}{1-p} \\
0 & \frac{2 \lambda q}{4-\lambda p}
\end{array}\right) \quad \text { and } \quad m_{2}=\left(\begin{array}{cc}
\lambda(1-p-q) & \frac{1-p-q}{1-p} \\
\frac{2 \lambda^{2}(1-p) q}{4-\lambda p} & \frac{2 \lambda q}{4-\lambda p}
\end{array}\right) ;
$$

$M$ is independent of $(a, b)$ if $\mathbb{P}(a>0, b>0)=1$.

PROOF. Using Skorohod's representation theorem (see [15]), with a change of the probability space we can assume that the sequences $\left(a_{n} / n\right)$ and $\left(b_{n} / n\right)$ converge almost surely (since they converge in $L_{1}$, they converge in distribution). 
If $\mathbb{P}(a>0, b>0)=1$ the state of the bin is $(2,1)$ at time 0 . If a new item 1 arrives, the bin is full and, during that time, the 2 in the bin is replaced by the initial items 2 . So, the state of the bin will come back to the state $(2,1)$ after an integrable duration of time (the time for an $M / M / 2$ queue starting with two customers to have only one customer). In this manner, a finite number of initial items 2 in the queue are served in the bin before a significant change occurs. If there is a departure when the state of the bin is $(2,1)$, another item 2 may enter if the item 1 leaves; otherwise an item 3 enters. Hence, after this event the state of the bin will be $(2,2)$ or $(3,1)$ with probability $1 / 2$. Since $\left(b_{n}\right)$ converges almost surely to infinity, there will be an item 2 in the queue with probability 1 at the occasion of such a departure. We conclude that the fact that an item 3 or a second item 2 enters the bin is independent of the the limit of $\left(a_{n}, b_{n}\right) / n$, as long as $a$ and $b$ are positive with probability 1 .

Throughout this discussion, we shall ignore small strings in our statements, that is, strings with an integrable length independent of the initial state. At the fluid level, most of them do not play a role (but some of them do play a role). As we already noticed:

Discussion. (A) With probability $1 / 2$ the item 2 leaves first. In this case the first item 3 enters the bin and then all the other $a_{n}-1$ items 3 will follow it in the bin. During that time, since $\lambda p<1$, items 1 are processed by the empty space in the bin. It is easily checked that the time $\tau_{1}$ to get rid of the initial items 3 is equivalent to $a_{n} \sim a n$.

At time $\tau_{1}$ the head of the queue is the original string of items 2 and 3 followed by another string of 2 and 3 built up during the service of items 3 . Consequently, using again the law of large numbers, the length of the queue is equivalent to $(b+\lambda(q+r) a) n$ (Lemma 16 of the Appendix). Very quickly an item of size 2 is in the bin. It is easy to check after an integrable amount of time that the state of the bin will be $(2,2)$. Starting from that time, all the initial items 2 are served consecutively: a string of items 3 builds up at the head of the queue followed by a shrinking string of 2's and 3's. At the end of the queue the new items arrived during that time form a string (since the bin is full, items 1 are not served during this phase). The time $\tau_{2}$ to serve all items 2 arrived before the state of the bin reaches $(2,2)$ is equivalent to the quantity

$$
(b+\lambda(q+r) a) \frac{q}{2(q+r)} n .
$$

At time $\tau_{1}+\tau_{2}$ there is a string of 3 's at the head of the queue of length equivalent to

$$
(b+\lambda(q+r) a) \frac{r}{q+r} n,
$$

followed by an i.i.d. string with distribution $F(d u)$ whose length is equivalent to the quantity

$$
\lambda(b+\lambda(q+r) a) \frac{q}{2(q+r)} n .
$$


If there is a departure of an item 2, it is immediately replaced by an item 2 or two items 1 ; items 3 cannot be served at that moment. Due to the i.i.d. structure of the queue at that time, it is then easily seen that after an integrable amount of time, the bin will be in the state $(1,1,1,1)$. From that time, all the 1 's will be served at rate 4 . The time $\tau_{3}$ it takes to empty the queue of items 1 and to have exactly a 2 and 1 in the bin is equivalent to

$$
\frac{\lambda p(b+\lambda(q+r) a) q}{2(q+r)(4-\lambda p)} n
$$

(take $\xi=\lambda p$ and $\mu=4$ in Lemma 16 of the Appendix). At time $\tau_{1}+\tau_{2}+\tau_{3}$ there is a string of 3's whose length is equivalent to (16), followed by a string of 2's and 3 's of length equivalent to

$$
\begin{aligned}
\lambda(b+ & \lambda(q+r) a) \frac{q}{2(q+r)}(q+r) n+\lambda(q+r) \frac{\lambda p(b+\lambda(q+r) a) q}{2(q+r)(4-\lambda p)} n \\
& =\frac{\lambda q}{2}(b+\lambda(q+r) a)\left(1+\frac{\lambda p}{4-\lambda p}\right)=\frac{2 \lambda q}{4-\lambda p}(b+\lambda(q+r) a) .
\end{aligned}
$$

For this case the distribution of $L(U)$ is given by $\mathbb{E}_{\mu_{n}}\left(R_{A_{n}, B_{n}, 0}(d x)\right)$ and $\left(A_{n}, B_{n}\right)$ satisfies the relation(14) with the matrix $M=m_{2}$.

The uniform integrability of the sequences $\left(Z_{n}\right)$ and $\left(n / Z_{n}\right)$ can be proved following the same discussion.

(B) With probability $1 / 2$ this is the item 1 ; another item 2 is in the bin, then all the other $b_{n}-1$ items 2 will be served. The method is the same as in the previous case. It is slightly simpler since the initial items 3 are not served at time $U_{1}$.

Finally, the discussion is similar on the set $\{a=0, b \neq 0\} \cup\{a \neq 0, b=0\}$; the difference is that the duration of some transitions described above are negligible in this case.

The next proposition gathers some facts and estimations which will be used in the sequel. Its proof, which is not difficult, follows the discussion of the above proof. It is skipped.

PROPOSITION 11. With the same notation as in Proposition 10, there exists a constant $K_{0}$ such that

$$
\limsup _{n \rightarrow+\infty} \mathbb{E}\left(\frac{U_{1}}{n}\right)<K_{0} \mathbb{E}(a+b) .
$$

If $a$ and $b$ are deterministic, positive and $Z_{n}=\left(A_{n}+B_{n}\right) /\left(a_{n}+b_{n}\right)$, the sequences $\left(Z_{n}\right)$ and $\left(1 / Z_{n}\right)$ are uniformly integrable.

The main result on the ultimate behavior of the fluid limits is contained in the following proposition. 
PROPOSITION 12. If $\left(M_{n}\right)$ is an i.i.d. sequence of random matrices with the same distribution as $M$ in Proposition 10 and $P_{n}=M_{n} \cdot M_{n-1} \cdots M_{1}$, there exist $\alpha, \beta>0$ and a function $\eta$ on $\mathbb{R}_{+}$such that for any $n \in \mathbb{N}$ and $x \in \mathbb{R}_{+}^{2}$,

$$
\mathbb{E}\left(\left\langle(\alpha, \beta), P_{n} \cdot x\right\rangle\right)=\eta(\lambda)^{n}\langle(\alpha, \beta), x\rangle,
$$

where $\langle\cdot, \cdot\rangle$ is the usual scalar product in $\mathbb{R}^{2}$. If

$$
\lambda^{*}=\frac{4-3 p-2 q-\sqrt{(4-3 p-2 q)^{2}-16 p(1-p-q)}}{2 p(1-p-q)},
$$

then $\eta(\lambda)<1$ if $\lambda<\lambda^{*}$ and $\eta(\lambda)>1$ if $\lambda^{*}<\lambda<4 / p$.

Proof. We denote by $\mathbb{E}\left(P_{n}\right)$ the expected value of the matrix $P_{n}$, that is, the matrix of the expected values of the coefficients of $P$. The i.i.d. property of the $M_{n}$ 's gives the relation $\mathbb{E}\left(P_{n}\right)=\mathbb{E}\left(M_{1}\right)^{n}$. The positive matrix $\mathbb{E}\left(M_{1}\right)$ has two positive eigenvalues. $\eta(\lambda)$ denotes the largest of them and $(\alpha, \beta)$ is the corresponding right eigenvector; $\alpha$ and $\beta$ can be chosen strictly positive (see, e.g., [31]). Consequently, we get

$$
\begin{aligned}
\mathbb{E}\left(\left\langle(\alpha, \beta), P_{n} \cdot x\right\rangle\right) & =\left\langle(\alpha, \beta), \mathbb{E}\left(P_{n}\right) \cdot x\right\rangle=\left\langle(\alpha, \beta), \mathbb{E}\left(M_{1}\right)^{n} \cdot x\right\rangle \\
& =\eta(\lambda)^{n}\langle(\alpha, \beta), x\rangle .
\end{aligned}
$$

It is easily seen that $\eta(\lambda)$ can be expressed as

$$
\eta(\lambda)=\max \left\{\frac{\left\langle\mathbb{E}\left(M_{1}\right), x\right\rangle}{\langle x, 1\rangle} \mid x \in \mathbb{R}_{+}^{2}\right\},
$$

since the components of $\mathbb{E}\left(M_{1}\right)$ are increasing with respect to $\lambda$ if $\lambda p<4$, the same property is true for the largest eigenvalue $\eta(\lambda)$. The smallest root of the equation $\eta(\lambda)=1$ is given by $\lambda=\lambda^{*}$. [Routine calculations show that the term under the square root in (20) is nonnegative if $p+q \leq 1$ and that $\lambda^{*} p<4$.] The proposition is proved.

COROLlary 13. With the notations of Proposition 12, if $\lambda<\lambda^{*}$ for any $\gamma>\eta(\lambda)$ and $x \in \mathbb{R}_{+}^{2}$ the sequence $\left(\gamma^{-n} P_{n} \cdot x\right)$ converges almost surely and in $L_{1}(\mathbb{P})$ to $(0,0)$.

Proof. Using (19) for $n=1$, it is easily seen that

$$
\left(Z_{n}\right)=\left(\left\langle(\alpha, \beta), \eta(\lambda)^{-n} P_{n} \cdot x\right\rangle\right)
$$

is a martingale. The sequence $\left(Z_{n}\right)$ being nonnegative, it converges almost surely to some finite limit $Z_{\infty}$. Since $\alpha$ and $\beta$ are positive and all the coefficients of $P_{n}$ are nonnegative, we deduce that the sequence $\left(\gamma^{-n} P_{n} \cdot x\right)$ converges almost surely to 0 for any $\gamma>\eta(\lambda)$. The $L_{1}$-convergence follows from the fact that 
$\mathbb{E}\left(P_{n} \cdot x\right)=\mathbb{E}\left(M_{1}\right)^{n} \cdot x$ and the fact that the eigenvalues of $\mathbb{E}\left(M_{1}\right)$ are in the interval $[0,1[$.

Our study here is simplified because all our matrices are nonnegative. For general results on the product of arbitrary random matrices, see [1], for example.

7. Ergodicity. Now we have all the necessary ingredients to get the ergodicity result. Starting from some arbitrary large initial state, the process "hits" some smooth random state whose size has the same order of magnitude of the initial state. From that time, under the appropriate condition, the Markov process shrinks with a factor $\gamma$ at each cycle described in the previous section. After some fixed number of such cycles, the size of the process will be a fraction of the original size. The ergodicity will be proved then.

THEOREM 14. When the arrival rate of the items is $\lambda$, the distribution of their sizes is given by

$$
F(d x)=p \delta_{1}+q \delta_{2}+(1-p-q) \delta_{3},
$$

and the size of the bin is 4 , if

$$
\begin{aligned}
& \lambda_{F F} \stackrel{\text { def }}{=} \min \left\{\frac{4-3 p-2 q-\sqrt{(4-3 p-2 q)^{2}-16 p(1-p-q)}}{2 p(1-p-q)},\right. \\
& \left.\frac{4}{3-2 p-q}\right\}
\end{aligned}
$$

then the Markov process $(X(t))$ describing the First Fit algorithm is ergodic when $\lambda<\lambda_{F F}$.

PROOF. If $\lambda p>1$, Proposition 4 shows that the condition $\lambda \mathbb{E}\left(S_{1}\right)<4$, that is, $\lambda(3-2 p-q)<4$ is sufficient for the ergodicity of $(X(t))$. One can check that in that case

$$
\frac{4}{3-2 p-q}<\frac{4-3 p-2 q-\sqrt{(4-3 p-2 q)^{2}-16 p(1-p-q)}}{2 p(1-p-q)} .
$$

We assume that conditions (21) and $\lambda p<1$ are satisfied. According to Theorem 1, to prove the ergodicity it is sufficient to prove that there exists a stopping time $V$ such that for any sequence $\left(x_{n}\right)=\left(b_{n}, l_{n}\right)$ of $s_{1}$ with $\left\|x_{n}\right\|=n$, the following inequalities hold:

$$
\limsup _{n \rightarrow+\infty} \frac{\mathbb{E}_{x_{n}}(\|X(V)\|)}{n} \leq 1-\varepsilon, \quad \limsup _{n \rightarrow+\infty} \frac{\mathbb{E}_{x_{n}}(V)}{n} \leq K,
$$


where $K>1$ and $\varepsilon>0$ are constants independent of the sequence $\left(x_{n}\right)$. The symbol $K$ for the constant is used throughout this proof; to avoid subscripts we keep the same letter.

According to Propositions 7 and 8 , if $X(0)=x_{n}$ there exists a stopping time $U_{0}$ such that:

(i) The distribution of $L\left(U_{0}\right)$ is given by $\mathbb{E}\left(R_{a_{n}, b_{n}, 0}(d x)\right)$, where $a_{n}$ and $b_{n}$ are some random variables and $B\left(U_{0}\right)=(2,1)$.

(ii) The following relations hold:

$$
\begin{gathered}
\limsup _{n \rightarrow+\infty} \frac{\mathbb{E}_{x_{n}} U_{0}}{n} \leq K, \\
\limsup _{n \rightarrow+\infty} \mathbb{E}_{x_{n}}\left(\frac{a_{n}+b_{n}}{n}\right)^{2} \leq \mathbb{E}_{x_{n}}\left(\frac{\left\|X\left(U_{0}\right)\right\|}{n}\right)^{2} \leq K .
\end{gathered}
$$

According to inequality (24), the sequence of random variables $\left(a_{n} / n, b_{n} / n\right)$ is tight for the convergence in distribution. By taking a subsequence, we can suppose that they jointly converge in distribution to some random variable $(a, b)$. Relation (24) shows that the sequence $\left(a_{n} / n, b_{n} / n\right)$ is uniformly integrable; consequently, it converges in $L_{1}$. In particular, the following convergence holds:

$$
\lim _{n \rightarrow+\infty} \mathbb{E}_{x_{n}}\left(\frac{a_{n}+b_{n}}{n}\right)=\mathbb{E}(a+b) \leq K
$$

The last inequality is a consequence of relation (24). Using again Skorohod's representation theorem (see [15]), with a change of the probability space we can assume that the sequences $\left(a_{n} / n\right)$ and $\left(b_{n} / n\right)$ converge almost surely to $a$ and $b$, respectively.

On the event $\left\{\left\|X\left(U_{0}\right)\right\| \leq\|X(0)\| / 4\right\}$ one sets $V=U_{0}$, so that

$$
\mathbb{E}_{X(0)}\left(\frac{\|X(V)\|}{\|X(0)\|} 1_{\left\{\left\|X\left(U_{0}\right)\right\| \leq\|X(0)\| / 4\right\}}\right) \leq \frac{1}{4} .
$$

We have to determine $V$ on the event $\left\{\left\|X\left(U_{0}\right)\right\|>\|X(0)\| / 4\right\}$. Proposition 10 shows that, on the event $\{a+b>0\}$ there exist a stopping time $U_{1}$, random variables $\left(A_{n, 1}\right),\left(B_{n, 1}\right)$ and a matrix $M_{1}$ independent of $(a, b)$ such that

$$
\mathbb{P}_{\mu_{n}}\left(B\left(U_{1}\right)=(2,1), L\left(U_{1}\right) \in d x\right)=\mathbb{E}_{\mu_{n}}\left(R_{A_{n, 1}, B_{n, 1}, 0}(d x)\right),
$$

where $\mu_{n}$ is the distribution of $X\left(U_{0}\right)$ when $X(0)=x_{n}$, and the relation

$$
\lim _{n \rightarrow+\infty} \frac{1}{n}\left(A_{n, 1}, B_{n, 1}\right)=M_{1} \cdot(a, b)
$$

holds almost surely and in $L_{1}$. 
From now on, until further notice, we work on the set $\{a+b>0\}$. We denote by $\left(\theta_{t} ; t \geq 0\right)$ the time-shift for the Markov process. If we iterate, we get the existence of random variables $\left(A_{n, 2}\right),\left(B_{n, 2}\right)$ and a matrix $M_{2}$ such that

$$
\begin{gathered}
\mathbb{P}_{X\left(U_{1}\right)}\left(B\left(U_{1} \circ \theta_{U_{1}}\right)=(2,1), L\left(U_{1} \circ \theta_{U_{1}}\right) \in d x\right) \\
=\mathbb{E}_{X\left(U_{1}\right)}\left(R_{A_{n, 2}, B_{n, 2}, 0}(d x)\right)
\end{gathered}
$$

and

$$
\lim _{n \rightarrow+\infty} \frac{1}{n}\left(A_{n, 2}, B_{n, 2}\right)=M_{2} M_{1}(a, b),
$$

almost surely and in $L_{1}$.

For $p \in \mathbb{N}$, we define the variable $U_{p+1}=U_{p}+U_{1} \circ \theta_{U_{p}} ; U_{p}$ is clearly a stopping time. Relation (27) gives the following equality:

$$
\mathbb{P}_{\mu_{n}}\left(B\left(U_{2}\right)=(2,1), L\left(U_{2}\right) \in d x\right)=\mathbb{E}_{\mu_{n}}\left(R_{A_{n, 2}, B_{n, 2}, 0}(d x)\right) .
$$

By induction, it is easily seen that there exist random variables $\left(A_{n, p}\right)$ and $\left(B_{n, p}\right)$, independent matrices $M_{p}, p \geq 2$ such that

$$
\mathbb{P}_{\mu_{n}}\left(B\left(U_{p}\right)=(2,1), L\left(U_{p}\right) \in d x\right)=\mathbb{E}_{\mu_{n}}\left(R_{A_{n, p}, B_{n, p}, 0}(d x)\right),
$$

and the convergence

$$
\lim _{n \rightarrow+\infty} \frac{1}{n}\left(A_{n, p}, B_{n, p}\right)=M_{p} M_{p-1} \cdots M_{2} M_{1}(a, b)
$$

holds almost surely and in $L_{1}$. According to Proposition 12,

$$
\lim _{n \rightarrow+\infty} \frac{1}{n}\left\langle(\alpha, \beta),\left(\mathbb{E}_{\mu_{n}}\left(A_{n, p}\right), \mathbb{E}_{\mu_{n}} B_{n, p}\right)\right\rangle=\gamma^{p}(\alpha a+\beta b) .
$$

Since $\left\|X\left(U_{p}\right)\right\|=2+A_{n, p}+B_{n, p}$,

$$
\begin{aligned}
& \limsup _{n \rightarrow+\infty} \frac{1}{n} \mathbb{E}_{\mu_{n}}\left(\left\|X\left(U_{p}\right)\right\|\right) \\
& \quad \leq \frac{1}{\alpha \wedge \beta} \lim _{n \rightarrow+\infty} \frac{1}{n}\left\langle(\alpha, \beta),\left(\mathbb{E}_{\mu_{n}}\left(A_{n, p}\right), \mathbb{E}_{\mu_{n}} B_{n, p}\right)\right\rangle \\
& \quad=\gamma^{p} \frac{\alpha \mathbb{E}(a)+\beta \mathbb{E}(b)}{\alpha \wedge \beta} \leq \gamma^{p} \frac{\alpha \vee \beta}{\alpha \wedge \beta} \mathbb{E}(a+b) \leq \gamma^{p} \frac{\alpha \vee \beta}{\alpha \wedge \beta} K,
\end{aligned}
$$

according to inequality (25). Since the condition $\lambda<\lambda_{F F}$ implies that $\gamma<1$, we choose $p \in \mathbb{N}$ such that

$$
\gamma^{p}<\frac{1}{4} \frac{\alpha \wedge \beta}{(\alpha \vee \beta) K}
$$


On the event $\left\{\left\|X\left(U_{0}\right)\right\|>\|X(0)\| / 4\right\}$, the variable $V$ is defined as $U_{0}+U_{p} \circ \theta_{U_{0}}$. For $n \in \mathbb{N}$, by the strong Markov property,

$$
\begin{aligned}
\mathbb{E}_{x_{n}}\left(\|X(V)\| 1_{\left\{\left\|X\left(U_{0}\right)\right\|>\left\|x_{n}\right\| / 4\right\}}\right) & =\mathbb{E}_{x_{n}}\left(\mathbb{E}_{\mu_{n}}\left(\left\|X\left(U_{p}\right)\right\|\right) 1_{\left\{\left\|X\left(U_{0}\right)\right\|>\left\|x_{n}\right\| / 4\right\}}\right) \\
& =\mathbb{E}_{x_{n}}\left(\mathbb{E}_{\mu_{n}}\left(A_{n, p}+B_{n, p}+2\right) 1_{\left\{\left\|X\left(U_{0}\right)\right\|>\left\|x_{n}\right\| / 4\right\}}\right) .
\end{aligned}
$$

We can assume that $\mathbb{P}(a+b=1 / 4)=0$; otherwise we replace the constant $1 / 4$ by some real $r$ less than $1 / 4$ such that $\mathbb{P}(a+b=r)=0$,

$$
\begin{aligned}
\left|\mathbb{E}_{x_{n}}\left(\mathbb{E}_{\mu_{n}}\left(\frac{\left\|X\left(U_{p}\right)\right\|}{n}\right)\left(1_{\left\{\left\|X\left(U_{0}\right)\right\|>\left\|x_{n}\right\| / 4\right\}}-1_{\{a+b>1 / 4\}}\right)\right)\right| \\
\leq C_{0} \mathbb{E}_{x_{n}}\left|1_{\left\{\left\|X\left(U_{0}\right)\right\|>\left\|x_{n}\right\| / 4\right\}}-1_{\{a+b>1 / 4\}}\right| \\
\quad+2 \mathbb{E}_{x_{n}}\left(\frac{\left\|X\left(U_{p}\right)\right\|}{n} 1_{\left\{\left\|X\left(U_{p}\right)\right\| / n \geq C_{0}\right\}}\right) .
\end{aligned}
$$

Due to the $L_{1}$-convergence of $\left\|X\left(U_{p}\right)\right\| / n=\left(A_{n, p}+B_{n, p}\right) / n$, the second term of the right-hand side is arbitrarily small uniformly on $n$ for some $C_{0}>0$. The first term converges to 0 since $\left\|X\left(U_{p}\right)\right\| / n$ converges almost surely to $a+b$ and $\mathbb{P}(a+b=1 / 4)=0$. Hence it is enough to consider the quantity

$$
\mathbb{E}_{x_{n}}\left(\mathbb{E}_{\mu_{n}}\left(\frac{\left\|X\left(U_{p}\right)\right\|}{n}\right) 1_{\{a+b>1 / 4\}}\right)
$$

Relation (28) implies that

$$
\limsup _{n \rightarrow+\infty} \mathbb{E}_{x_{n}}\left(\frac{\|X(V)\|}{n} 1_{\left\{\left\|X\left(U_{0}\right)\right\|>\left\|x_{n}\right\| / 4\right\}}\right) \leq \gamma^{p} \frac{\alpha \vee \beta}{\alpha \wedge \beta} K \leq \frac{1}{4} .
$$

Inequalities (23) and (18) show that there exists some constant $K$ such that

$$
\limsup _{n \rightarrow+\infty} \frac{\mathbb{E}_{x_{n}}(V)}{\|X(0)\|} \leq K
$$

and relations (26) and (29) give

$$
\limsup _{n \rightarrow+\infty} \mathbb{E}_{x_{n}}\left(\frac{\|X(V)\|}{\|X(0)\|}\right) \leq \frac{1}{2}
$$

The proof of the proposition is complete.

REMARK. The independence of the matrices $M_{2}, M_{3}, \ldots$ has been used on the proof. Since one may have $a=0$ or $b=0, M_{1}$ is not necessarily independent of $(a, b)$. It is nevertheless true after step 2 for $M_{p}$, for $p \geq 2$ (see the definition of $m_{1}$ and $m_{2}$ in Proposition 10). 


\section{Transience.}

THEOREM 15. When the arrival rate of the items is $\lambda$, the distribution of their sizes is given by $F(d x)=p \delta_{1}+q \delta_{2}+(1-p-q) \delta_{3}$, and the size of the bin is 4 . The Markov process $(X(t))$ describing the First Fit algorithm is transient when $\lambda>\lambda_{F F}$, where $\lambda_{F F}$ is defined by (21).

ProOF. We assume that the initial distribution of $(L(t))$ is given by $R_{a, b, 0}$ and the initial state of the bin is $(2,1)$.

With the notation of Proposition 10, $U_{1}$ is the first time when all the initial items 2 have left the queue, the items 2 in the bin have been served and the state of the bin is $(2,1)$. As in the proof of Proposition 14, we define the sequence of stopping times $\left(U_{p}\right)$ by

$$
U_{p+1}=U_{p}+U_{1} \circ \theta_{U_{p}} .
$$

The variable $U_{p+1}$ is the first moment when all the items 2 present at time $U_{p}$ have left the queue and the state of the bin is $(2,1)$. Clearly enough, the sequence $\left(L\left(U_{p}\right)\right)$ is a homogeneous irreducible Markov chain on $\mathcal{T}^{(\mathbb{N})}$.

The distribution of $X\left(U_{1}\right)$ is represented by

$$
\mathbb{P}_{R_{a, b, 0}}\left(X\left(U_{1}\right) \in d x\right)=\mathbb{E}\left(R_{A_{a, b}, B_{a, b}, 0}(d x)\right) ;
$$

almost surely $U_{1}$ is a finite stopping time. Propositions 10 and 12 show that there exist constants $\alpha, \beta$ such that

$$
\lim _{a+b \rightarrow+\infty} \frac{\alpha A_{1}+\beta B_{1}}{\alpha a+\beta b}=\gamma>1
$$

almost surely.

We assume that the Markov process $(X(t))$ is recurrent. In particular it visits the state $y_{0}=(\varnothing,(2,1))$ infinitely often; that is, with probability 1 the queue will be empty and the state of the bin will be $(2,1)$. The first time the process $(X(t))$ visits the state $y_{0}$ is necessarily at one of the moments $U_{p}, p \geq 1$. Consequently, the Markov chain $\left(L\left(U_{p}\right)\right)$ visits the state $y_{0}$ with probability 1 .

We now define a Lyapounov function on the state space of $\left(L\left(U_{p}\right)\right)$ by

$$
f(l)=\log (1+\alpha p+\beta(\|l\|-p)),
$$

if $l=\left(l_{i}\right)$ and $p=\inf \left\{k-1 / l_{k} \neq 2\right\}$. With the notations defined above, we have $f\left(L\left(U_{1}\right)\right)=\log \left(1+\alpha A_{a, b}+\beta B_{a, b}\right)$; consequently,

$$
\mathbb{E}_{R_{a, b, 0}}\left(f\left(L\left(U_{1}\right)\right)-f\left(L\left(U_{0}\right)\right)\right)=\mathbb{E}_{R_{a, b, 0}}\left(\log \left(\frac{1+\alpha A_{a, b}+\beta B_{a, b}}{1+\alpha a+\beta b}\right)\right) .
$$

According to Proposition 10, the random variables

$$
\left(1+\alpha A_{a, b}+\beta B_{a, b}\right) /(1+\alpha a+\beta b)
$$


and their inverse are uniformly integrable. Consequently, the elementary inequality

$$
|\log x| \leq x+\frac{1}{x}
$$

for $x>0$, convergence (30) and Lebesgue's theorem show that

$$
\lim _{a+b \rightarrow+\infty} \mathbb{E}_{R_{a, b, 0}}\left(f\left(L\left(U_{1}\right)\right)-f\left(L\left(U_{0}\right)\right)\right)=\log \gamma>0 .
$$

Hence there exists some constant $K_{0}$ such that if $a+b \geq K_{0}$,

$$
\mathbb{E}_{R_{a, b, 0}}\left(f\left(L\left(U_{1}\right)\right)-f\left(L\left(U_{0}\right)\right)\right) \geq(\log \gamma) / 2 .
$$

In the same way, the following inequality holds:

$$
\begin{aligned}
& \mathbb{E}_{R_{a, b, 0}}\left(\left|f\left(L\left(U_{1}\right)\right)-f\left(L\left(U_{0}\right)\right)\right|^{2}\right) \\
& \quad \leq 6 \log (\alpha \vee \beta)^{2}+4 \mathbb{E}_{R_{a, b, 0}}\left(\log ^{2}\left(\frac{1+A_{a, b}+B_{a, b}}{1+\alpha a+\beta b}\right)\right) .
\end{aligned}
$$

The elementary inequality

$$
\log ^{2} x \leq \frac{4}{e^{2}}\left(x+\frac{1}{x}\right)
$$

for $x>0$ and the uniform integrability argument give the following inequality:

$$
\sup _{a, b: a+b>K} \mathbb{E}_{R_{a, b, 0}}\left(\left|f\left(L\left(U_{1}\right)\right)-f\left(L\left(U_{0}\right)\right)\right|^{2}\right)<+\infty .
$$

A theorem by Lamperti [23] (see [16] or [26]) states that if relations (32) and (31) are satisfied, then the Markov chain $\left(L\left(U_{p}\right)\right)$ is transient. In particular this implies that there exists an initial state such that the chain will never visit the state $y_{0}$ with positive probability. This contradicts our assumption on the recurrence of $(X(t))$. The theorem is proved.

The case of symmetrical distributions. The distribution $F$ is symmetrical if

$$
F(d x)=p \delta_{1}+(1-2 p) \delta_{2}+p \delta_{3},
$$

for $p \in[0,1 / 2]$. Since the expected value of the size of the items is $1 / 2$ for all these distributions, the value $\lambda_{F F}$ of the corresponding critical $\lambda$ cannot exceed 2 .

According to Theorem 14 the critical value of $\lambda$ for the First Fit algorithm is given by

$$
\lambda_{F F}=\frac{2+p-\sqrt{4+4 p-15 p^{2}}}{2 p^{2}}
$$

and for the FIFO policy, it is given by (see [21])

$$
\lambda_{\mathrm{FIFO}}=\frac{12}{6+12 p-24 p^{2}+10 p^{3}-p^{4}} .
$$




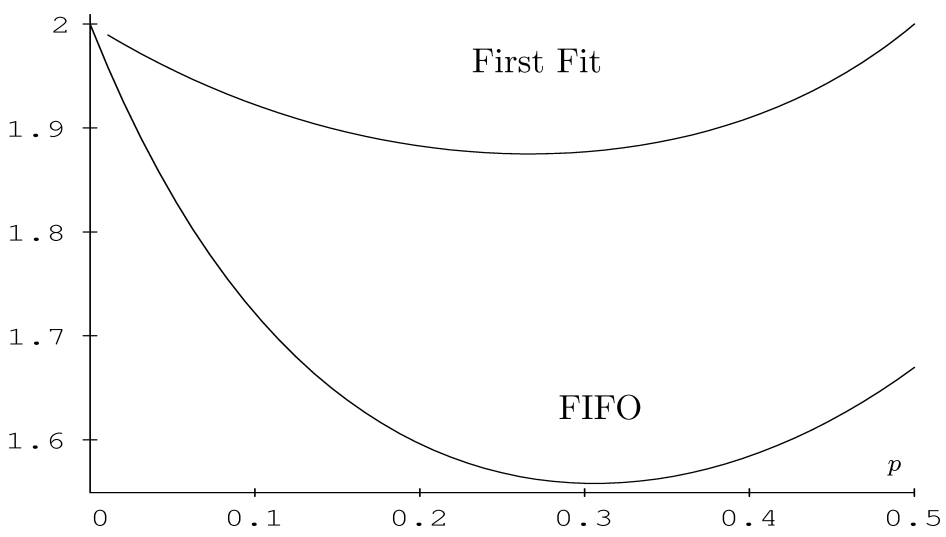

FIG. 2. The effective bandwidth of the FIFO and First Fit policies for symmetrical distributions on $\{1,2,3\}$.

Coffman and Stolyar [8] considered the stability of the algorithms First Fit and Best Fit when the services are constant equal to 1 . In that setting they have proved that the natural condition $\lambda E\left(S_{1}\right)<C$ is sufficient for the stability when the variable $S_{1}$ is uniformly distributed on $\{1 / n, 2 / n, \ldots,(n-1) / n\}$. This property is not true when the services are exponentially distributed (see Figure 2 ).

Figure 3 shows that the First Fit algorithm is much more efficient than the FIFO policy. This comparison is intuitive since the First Fit algorithm reduces the wasted space in the bin. The minimal value of $\lambda_{F F}$ is $15 / 8=1.875$ which is close to 2 , the optimal value. Notice that in the case where there are only 1's and 3's equally likely, $\lambda_{F F}$ is 2 , hence is optimal; this is not the case for the FIFO policy.

The fact that, even in the case of symmetrical distributions, the constant $\lambda_{c}$ is not $1 / \mathbb{E}\left(S_{1}\right)=2$ in general can be (roughly) explained as follows. The only time

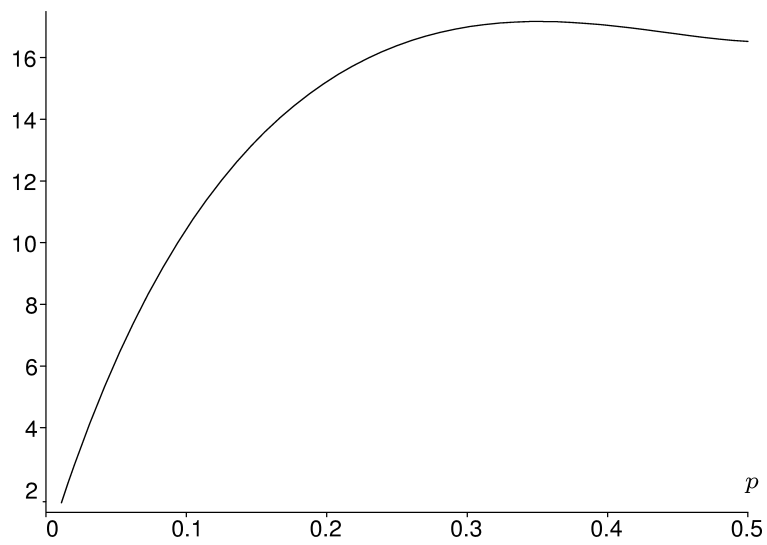

FIG. 3. First Fit compared to FIFO for symmetrical distributions on $\{1,2,3\}$ : Percentage of increase of the effective bandwidth. 
when there is some potentially wasted space is when an item 3 is in the bin. During that time some of the items 1 are served in the empty space left by the 3's. If all of them were served in this way, it is easy to see that it would imply $\lambda_{c}=2$. This is not the case in fact. Indeed, in the description of the cycle of the previous section, we have seen that during some time, four items 1 are in the bin. In particular these items 1 will not help to fill the empty spaces left by the 3 's. Consequently, the condition $\lambda<2$ is, in general, not sufficient to ensure ergodicity.

\section{APPENDIX}

The following elementary lemma is constantly used (some times implicitly) in Sections 4 and 6.

LEMMA 16. If $\mathcal{N}_{\xi}, \mathcal{N}_{\mu}$ are independent Poisson processes on $\mathbb{R}_{+}$with respective parameters $\xi, \mu$ with $0<\xi<\mu$, and $(v(t))$ is a cadlag (i.e., right continuous functions having a limit on the left at any point), nondecreasing process on $\mathbb{R}_{+}$, independent of these Poisson processes, such that $(v(t) / t)$ converges to $v$ almost surely and in $L_{1}$, then

$$
\left.\left.Z(t)=\frac{1}{t}\left(\left(\mathcal{N}_{\xi}-\mathcal{N}_{\mu}\right)(] 0, v(t)\right]\right)\right)-(\xi-\mu) v
$$

converges to 0 almost surely and in $L_{1}$. If

$$
\left.\left.\tau(t)=\inf \left\{s \geq 0 \mid v(t)+\left(\mathcal{N}_{\xi}-\mathcal{N}_{\mu}\right)(] 0, s\right]\right) \leq 0\right\},
$$

then $\tau(t) / t$ converges to $v /(\mu-\xi)$ almost surely and in $L_{1}$.

PROOF. To prove the first part, it is enough to show that

$$
\left.\left.Z_{1}(t)=\frac{1}{t}\left(\left(\mathcal{N}_{\xi}-\mathcal{N}_{\mu}\right)(] 0, v(t)\right]\right)-(\xi-\mu) v(t)\right)
$$

converges to 0 almost surely and in $L_{1}$. For $t \geq 0$, using the independence properties, we get

$$
\begin{aligned}
\mathbb{E}\left(Z_{1}(t)^{2}\right) & \left.\left.=\frac{1}{t^{2}} \int_{0}^{+\infty} \mathbb{E}\left(\left(\left(\mathcal{N}_{\xi}-\mathcal{N}_{\mu}\right)(] 0, x\right]\right)-(\xi-\mu) x\right)^{2}\right) \mathbb{P}(v(t) \in[x, x+d x]) \\
& =\frac{1}{t^{2}} \int_{0}^{+\infty}(\xi+\mu) x \mathbb{P}(v(t) \in[x, x+d x])=(\xi+\mu) \frac{\mathbb{E}(v(t))}{t^{2}},
\end{aligned}
$$

hence the convergence to 0 in $L_{1}$.

On the set $\{v>0\}$, the law of large numbers for Poisson processes gives immediately the almost sure convergence of $(Z(t))$. If $v>0$, for $\varepsilon>0$ and $t$ sufficiently large we have $v(t) \leq \varepsilon t$, hence

$$
\left.\left.|Z(t)| \leq \frac{1}{t}\left(\left(\mathcal{N}_{\xi}+\mathcal{N}_{\mu}\right)(] 0, \varepsilon t\right]\right)\right) ;
$$


consequently,

$$
\limsup _{t \rightarrow+\infty}|Z(t)| \leq(\xi+\mu) \varepsilon .
$$

We get the almost sure convergence on $\{v=0\}$ by letting $\varepsilon$ go to 0 .

Using the law of large numbers for Poisson processes, the almost sure convergence of $\tau(t) / t$ to $v /(\mu-x i)$ is straightforward to obtain. By definition of $\tau(t)$ we have,

$$
\left.\left.\left.\left.v(t)+\left(\mathcal{N}_{\xi}-\mathcal{N}_{\mu}\right)(] 0, \tau(t)\right]\right) \leq 0 \leq v(t)+\left(\mathcal{N}_{\xi}-\mathcal{N}_{\mu}\right)(] 0, \tau(t)\right]\right)+1 .
$$

Taking the expected value of this inequality and using Wald's formula, we obtain,

$$
\frac{1+\mathbb{E}(v(t))}{t} \geq(\mu-\xi) \frac{\mathbb{E}(\tau(t))}{t} \geq \frac{\mathbb{E}(v(t))}{t} .
$$

Hence $\mathbb{E}(\tau(t)) / t$ converges to $\mathbb{E}(v) /(\mu-\xi)$; this is enough to ensure the $L_{1}$ convergence of $(\tau(t) / t)$ since it already converges almost surely. The lemma is proved.

\section{REFERENCES}

[1] Bougerol, P. and LacroiX, J. (1985). Products of Random Matrices with Applications to Schrödinger Operators. Birkhäuser, Boston.

[2] Bramson, M. (1994). Instability of FIFO queueing networks. Ann. Appl. Probab. 4 414-431.

[3] BRAmSON, M. (1994). Instability of FIFO queueing networks with quick service times. Ann. Appl. Probab. 4 693-718.

[4] BRAMSON, M. (1996). Convergence to equilibria for fluid models of FIFO queueing networks. Queueing Systems Theory Appl. 22 5-45.

[5] Chen, H. and Mandelbaum, A. (1991). Discrete flow networks: bottleneck analysis and fluid approximations. Math. Oper. Res. 16 408-446.

[6] Coffman, E. G. JR. and Lueker, G. S. (1991). Probabilistic Analysis of Packing and Partitioning Algorithms. Wiley, New York.

[7] Coffman, E. G., Feldman, A., Kahale, N. and Poonen, B. (1997). Computing call admission capacities in linear networks. Preprint.

[8] Coffman, JR., E. G. and Stolyar, A. L. (2001). Bandwidth packing. Algorithmica 29 $70-88$.

[9] DAI, J. G. (1995). On positive Harris recurrence of multiclass queueing networks: a unified approach via fluid limit models. Ann. Appl. Probab. 5 49-77.

[10] DAI, J. G. (1996). A fluid limit model criterion for instability of multiclass queueing networks. Ann. Appl. Probab. 6 751-757.

[11] Dantzer, J.-F., Haddani, M. and Robert, P. (2000). On the stability of a bandwidth packing algorithm. Probab. Engrg. Inform. Sci. 14 57-79.

[12] Dumas, V. (1997). A multiclass network with non-linear, non-convex, non-monotonic stability conditions. Queueing Systems Theory Appl. 25 1-43.

[13] Dumas, V. (1999). Diverging paths in FIFO fluid networks. IEEE Trans. Automat. Control 44 191-194.

[14] Dupuis, P. and Williams, R. J. (1994). Lyapounov functions for semimartingale reflecting Brownian motions. Ann. Probab. 22 680-702. 
[15] Ethier, S. N. and Kurtz, T. G. (1986). Markov Processes: Characterization and Convergence. Wiley, New York.

[16] Fayolle, G., Malyshev, V. A. and Men'shikov, M. V. (1995). Topics in the Constructive Theory of Countable Markov Chains. Cambridge Univ. Press.

[17] Filonov, Y. (1989). A criterion for the ergodicity of discrete homogeneous Markov chains. Ukrain. Mat. Zh. 41 1421-1422.

[18] GaǏrat, A. S., Malyshev, V. A., Men'shikov, M. V. and Pelikh, K. D. (1995). Classification of Markov chains describing the evolution of random strings. Russian Math. Surveys 50 237-255.

[19] HAS'Minskil̆, R. Z. (1980). Stochastic Stability of Differential Equations. Sijthoff and Noordhoff, Alphen aan den Rijn. (Transl. from Russian).

[20] Hirsch, M. W. and Smale, S. (1974). Differential Equations, Dynamical Systems, and Linear Algebra. Academic Press, New York.

[21] Kipnis, C. and Robert, P. (1990). A dynamic storage process. Stochastic Process. Appl. 34 155-169.

[22] Kumar, P. R. and Seidman, T. I. (1990). Dynamic instabilities and stabilization methods in distributed real-time scheduling of manufacturing systems. IEEE Trans. Automat. Control 35 289-298.

[23] LAMPERTI, J. (1960). Criteria for the recurrence or transience of stochastic process I. J. Math. Anal. Appl. $1314-330$.

[24] LU, S. H. and Kumar, P. R. (1991). Distributed scheduling based on due dates and buffer priorities. IEEE Trans. Automat. Control 36 1406-1416.

[25] Malyshev, V. A. (1994). Stabilization laws in the evolution of a random string. Problems Inform. Transmission 30 79-95.

[26] Meyn, S. and Tweedie, R. (1993). Markov Chains and Stochastic Stability. Springer, New York.

[27] Meyn, S. P. (1995). Transience of multiclass queueing networks via fluid limit models. Ann. Appl. Probab. 5 946-957.

[28] Pukhal'skil̆, A. A. and RYBKo, A. N. (2000). Nonergodicity of queueing networks when their fluid models are unstable. Problemy Peredachi Informatsii 36 26-46.

[29] Robert, P. (2002). Smooth initial distributions and fluid limits for multi-class queueing systems. Unpublished manuscript.

[30] RybKo, A. N. and Stolyar, A. L. (1992). On the ergodicity of random processes that describe the functioning of open queueing networks. Problems Inform. Transmission $\mathbf{2 8}$ 3-26.

[31] Seneta, E. (1981). Nonnegative Matrices and Markov Chains, 2nd ed. Springer, New York.

[32] SERFOzO, R. (1999). Introduction to Stochastic Networks. Springer, New York.

DÉPARTEMENT DE MATHÉMATiques

BÂTIMENT FERMAT

UNIVERSITÉ DE VERSAILLES-ST-QUENTIN

45, AVENUE DEs ÈtATS-UNIS

78035 VERSAILLES

FRANCE

E-MAIL: dantzer@ fermat.math.uvsq.fr
INRIA

Domaine De Voluceau

B.P. 105

78153 LE ChesNay CEDEX

FRANCE

E-MAIL: Philippe.Robert@inria.fr 